\title{
Retinal Nerve Fibre Layer Thickness Changes after Pan-Retinal Photocoagulation in Diabetic Retinopathy
}

\author{
Goh SY, Ropilah AR, Othmaliza O, Mushawiahti M ( $\bowtie)$ \\ Department of Ophthalmology, Faculty of Medicine, Universiti Kebangsaan Malaysia Medical Centre, \\ Jalan Yaacob Latif, Bandar Tun Razak, 56000 Cheras, Kuala Lumpur, Malaysia.
}

\begin{abstract}
Diabetic retinopathy is a disease involving microangiopathic changes in response to chronic hyperglycaemia and pan retinal photocoagulation (PRP) is currently the mainstay of treatment for proliferative retinopathy. In the present study, we evaluated the effect of pan retinal photocoagulation (PRP) on retinal nerve fibre layer (RNFL) thickness in patients with diabetic retinopathy using optical coherence tomography (OCT). This was a prospective longitudinal study. Patients with Type 2 diabetes mellitus with proliferative diabetic retinopathy (PDR) or very severe non(N)PDR requiring laser treatment were included in the study. PRP was performed by a single trained personnel. Peripapillary RNFL located $3.4 \mathrm{~mm}$ around the optic disc was evaluated using time-domain OCT. Examination was performed before treatment, and 2 and 4 months after laser treatment. In total, 39 subjects (39 eyes) were recruited into this study. Twenty-nine patients had PDR and 10 had very severe NPDR. Mean age was $54.97 \pm 8.38$ years. Male and female genders were almost equally distributed with 18 males and 21 females. Median thickness of average RNFL at baseline was 108.8 um (interquartile range [IQR] 35.3). At two months post-procedure, average RNFL thickness significantly increased to 117.4 (IQR 28.6; $\mathrm{P}=0.006$ ). Although, other quadrants revealed a similar trend of increasing thickness at two months but it was not significant. At 4 months post-laser treatment, RNFL thickness in all quadrants reduced to baseline levels with insignificant changes of thickness compared to prior to laser treatment. There was also no significant association between changes in RNFL thickness and HbA1c levels (P $=0.77$ ). In conclusion, PRP causes transient thickening of the RNFL which recovers within 4 months post-laser treatment. At the same time, poor sugar control has no direct influence on the RNFL changes after PRP.
\end{abstract}

Keywords: diabetic retinopathy, light coagulation, optical coherence, retinal ganglion cells, tomography

\section{Correspondence:}

Mushawiahti Mustapha, Department of Ophthalmology, Faculty of Medicine, Universiti Kebangsaan Malaysia Medical Centre, Jalan Yaacob Latif, Bandar Tun Razak, 56000 Cheras, Kuala Lumpur, Malaysia. Tel: +60391455981 Fax: +60391456673

Email: drmusha@gmail.com/drmusha@yahoo.com

\section{Introduction}

Diabetic retinopathy is a disease involving microangiopathic changes in response to chronic hyperglycaemia. It is characterized by progressive dysfunction of retinal microvasculature, including microvascular occlusion and microvascular leakage (1). Successful management of diabetic retinopathy via the combination of glucose control, laser therapy, and vitrectomy represents one of the most striking achievements of modern ophthalmology. Routine fundus examinations initiated prior to the development of significant retinopathy, based on the recommendations by the Early Treatment Diabetic Retinopathy Study (ETDRS), lowers the risk of severe visual loss to less than 5\% (2). Pan-retinal photocoagulation (PRP) is currently the mainstay of treatment for proliferative retinopathy $(3,4)$.

The therapeutic effects of PRP rely on the destruction of the outer retina, which helps reduce oxygen demand while maintaining a constant oxygen input. Laser burns may also serve as physiological windows through which oxygen diffuses from the choroid, 
increasing vitreous oxygenation and induced vasoconstriction (3). The energy of a conventional argon laser is transformed to heat at the level of the retinal pigment epithelium (RPE) and the outer retinal layer. However, longer laser wavelengths will penetrate deeper than the RPE and cause more destructive changes to the retina, RPE, and choriocapillaris (5).

Optical coherence tomography (OCT) is a modern biomicroscopic imaging technique widely used to obtain high-resolution cross sectional images of the retinal layers. It resembles an optical biopsy and provides images in real time. OCT assists clinicians in observing macular changes as well as assessing the status of optic nerve damage in glaucoma by looking at peripapillary retinal nerve fibre layer (RNFL) thickness (6). Therefore, it is important for the clinician to be aware of conditions that predispose to abnormal RNFL thickness, such as PRP.

Many studies have reported variable findings with respect to possible changes in the RNFL following PRP $(5,7)$. For instance, Lim et al. in a cross sectional study, described significant thinning of the inferior and nasal RNFL in post-PRP eyes (7). Conversely, in another retrospective study by Kim and Cho in the Korean Journal of Ophthalmology, reported that there was no significant relationship between treatment and the control group (5). The present study aimed to longitudinally evaluate peripapillary RNFL changes after PRP in our populationusing the OCT which is more objective and to look at the possible association between RNFL and blood sugar control, in particular HbA1c levels.

\section{Materials and Methods}

This is a prospective longitudinal study conducted in Universiti Kebangsaan Malaysia Medical Center (UKMMC), Kuala Lumpur, Malaysia. It was conducted between July 2010 and May 2013. The participants of this study were patients diagnosed with proliferative diabetic retinopathy (PDR) and very severe non-(N) PDR who required laser treatment. An experienced ophthalmologist graded the stages of diabetic retinopathy clinically and diagnosis was made based on the ETDRS classification recommendation. Ethical approval was obtained from the Research and Ethical Committee of UKMMC.

Inclusion criteria were: patients with type 2 diabetes mellitus with very severe NPDR (ETDRS classification) or PDR, clear media without clinically significant cataract, age of diagnosis of diabetes mellitus $>35$ years of age and good signal strength of more than five on time-domain OCT. Exclusion criteria were Type 1 diabetes mellitus, media opacity, such as significant ocular media complications (which precluded good OCT images), eyes with co-existing glaucomatous optic disc changes, previous surgery, degenerative myopia, uveitis, and other retinal vascular pathology that may affect RNFL thickness. Written consent was obtained from all participants. Examination involved visual acuity, anterior segment evaluation, fundus photography, and peripapillary RNFL evaluation using time-domain OCT (Carl Zeiss Meditec Inc., Dublin, CA, USA). HbA1c levels of every patient were also recorded.

PRP was performed by single trained personnel. An argon green $532 \mathrm{~nm}$ (Ellex SOLITAIRE, FL, USA) laser machine was used. A total of 900 to 1200 laser spots were required for each setting. Spot size was 200 $\mu \mathrm{m}$ and pulse duration 100 milliseconds. Power was adjusted to attain grey burns and spots were placed one spot distance apart. Repeated PRP lasering was performed in two to four sessions until the new vessels regressed. Fundus was reviewed by another qualified observer to determine the sufficiency of treatment. Intervals between the laser sessions were fixed at 1 to 2 weeks. The total laser spots over the entire treatment period ranged from 2,000 to 2,500 shots. Repeat OCT was performed 2 and 4 months after treatment.

Peripapillary RNFL measurements were obtained using optical coherence tomography (Stratus OCT3, Carl Zeiss Meditech). Through a dilated pupil, a 3.4 mm diameter ring was centred around the optic nerve head and 768 A-scans were acquired using the fast RNFL thickness protocol. The peripapillary regions assessed included temporal $\left(316^{\circ}-45^{\circ}\right)$, superior $\left(46^{\circ}-\right.$ $\left.135^{\circ}\right)$, nasal $\left(136^{\circ}-225^{\circ}\right)$, and inferior $\left(226^{\circ}-315^{\circ}\right)$. In clock-hour positions, 3 o'clock was considered temporal; 6 o'clock, inferior; 9 o'clock, nasal; and 12 o'clock, superior. The average thickness $\left(360^{\circ}\right)$ was also measured.

\section{Statistical Analysis}

Statistical analyses in this study were presented descriptively. Univariate, bivariate, and multivariate analyses were performed using the Statistical Package for Social Sciences (SPSS) version 20.0 (IBM Corp, Armonk, NY, USA). Paired t-test was used for normal data and Wilcoxon sign ranked test for data not normally distributed. A P-value of less than 0.05 was considered statistically significant. 
Table 1: Summary of the demographic data and clinical characteristics of the study population

\begin{tabular}{lcc}
\hline Variable & $\begin{array}{c}\text { Patients } \\
\mathbf{N ~ ( N ~ = ~ 3 9 ) ~}\end{array}$ & $\begin{array}{c}\text { Percentage } \\
\text { (\%) }\end{array}$ \\
\hline Age (Years) (n, \%) & & \\
Mean (SD) & $54.97 \pm 8.381$ & \\
Gender (n, \%) & & 46.2 \\
Male & 18 & 53.8 \\
Female & 21 & \\
Ethnicity (n, \%) & & 59.0 \\
Malay & 23 & 35.9 \\
Chinese & 14 & 5.1 \\
Indian & 2 & 10.3 \\
HbA1c level (n, \%) & & 43.6 \\
$<6.5 \%$ & 4 & 23.1 \\
6.5- 8.0\% & 17 & 23.1 \\
8.0-10.0\% & 9 & \\
$>10.0 \%$ & 9 & 25.6 \\
Diabetic retinopathy type (n, \%) & & \\
PDR & 29 & \\
Very severe NPDR & 10 & \\
\hline
\end{tabular}

Table 2: Peripapillary RNFL thickness for each quadrant pre- and post-PRP treatment. Baseline value was RNFL thickness measured prior to PRP treatment and followed by the RNFL thickness measurements 2 and 4 months after PRP treatment

\begin{tabular}{lccccc}
\hline \multirow{2}{*}{ Peripapillary area } & \multicolumn{3}{c}{$\begin{array}{c}\text { RNFL thickness } \\
\text { (median } \pm \text { interquantile range, } \boldsymbol{\mu m} \text { ) }\end{array}$} & \multicolumn{2}{c}{$\boldsymbol{P}$-value } \\
\cline { 2 - 6 } & Baseline & $\mathbf{2}$ months & $\mathbf{4}$ months & $\boldsymbol{P}_{\text {-value }} \mathbf{1}^{\mathbf{1}}$ & $\boldsymbol{P}_{\text {-value }} \mathbf{2}^{2}$ \\
\cline { 2 - 6 } Superior $(\mu \mathrm{m})$ & $138.0 \pm 37.0$ & $140.0 \pm 33.0$ & $137.0 \pm 29.0$ & 0.157 & 0.46 \\
Nasal $(\mu \mathrm{m})$ & $77.0 \pm 38.0$ & $84.0 \pm 26.0$ & $77.0 \pm 24.0$ & 0.663 & 0.75 \\
Inferior $(\mu \mathrm{m})$ & $139.0 \pm 50.0$ & $137.0 \pm 44.0$ & $133.0 \pm 40.0$ & 0.057 & 0.23 \\
Temporal $(\mu \mathrm{m})$ & $81.0 \pm 23.0$ & $89.0 \pm 24.0$ & $87.0 \pm 27.0$ & 0.017 & 0.06 \\
Average $(\mu \mathrm{m})$ & $108.8 \pm 35.3$ & $117.4 \pm 28.6$ & $111.0 \pm 30.8$ & 0.006 & 0.065 \\
\hline
\end{tabular}

P-value1: for the first interval; from baseline to month 2;

P-value2: for the second interval; from baseline to month 4;

Significant when $\mathrm{P}<0.05$,

\section{Results}

\section{Demographics}

A total of 50 eligible subjects were initially recruited at the beginning of the study. Only one eye from each subject was chosen. However, 11 PDR subjects had to be excluded during the study due to development of vitreous haemorrhage (3), poor OCT signal (2), defaulted follow-up (5), and deceased (1). Only 39 subjects completed the review and were analysed at the end of the study. In total, 29 patients had PDR and 10 patients had very severe non-PDR. Mean age was
$54.97 \pm 8.38$ years, and there were 18 males and 21 females. The majority subjects had suboptimal sugar control, as $\mathrm{HbA1c}$ was within the range of $6.5 \%$ to $8.0 \%$ and this accounted for $43.6 \%$ of the recruited subjects. The remaining $46.2 \%$ presented with HbA1c levels of $>8.0 \%$ (Table 1 ).

\section{Peripapillary Retinal Nerve Fibre Layer Thickness}

The subjects were not normally distributed; nonparametric analysis was used. Baseline RNFL thickness according to quadrant were: Superior $138.0 \pm 37.0 \mu \mathrm{m}$, nasal $77.0 \pm 38.0 \mu \mathrm{m}$, inferior $139.0 \pm 50.0 \mu \mathrm{m}$, and 


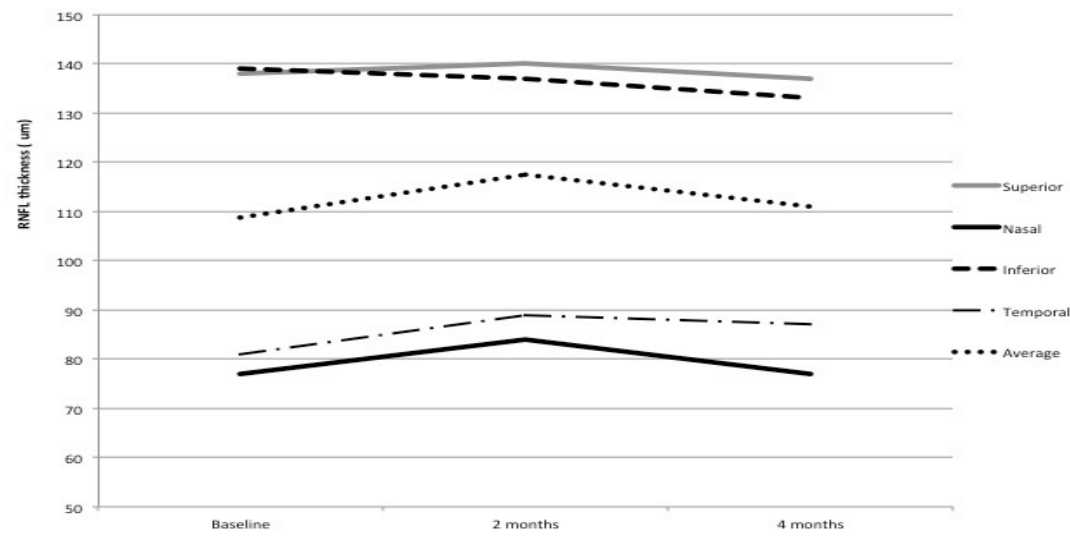

Figure 1: Peripapillary RNFL thickness changes (according to quadrant) before and after argon laser treatment. Transient thickening occurred month 2 post-treatment. Normalisation of the RNFL in all quadrants at 4 months.

temporal $81.0 \pm 23.0 \mu \mathrm{m}$, with an overall average of $108.8 \pm 35.3 \mu \mathrm{m}$. There was an increase in the average RNFL thickness two months post-treatment.

Median thickness for average RNFL was $108.8 \mu \mathrm{m}$ (interquartile range [IQR] 35.3) at baseline, which increased to $117.4 \mu \mathrm{m}$ (IQR 28.6) after treatment $(\mathrm{P}=$ 0.006). However, there was mild decrease in average RNFL thickness from $117.4 \mu \mathrm{m}$ (IQR 28.6) at 2 months to $111.0 \mu \mathrm{m}$ (IQR 30.8) at 4 months, which was not statistically significant $(P=0.065)$. The overall average RNFL change from baseline to 4 months was also not statistically significant, $\mathrm{P}=0.65$. Baseline superior RNFL thickness, and at month 2 and month 4 was $138.0 \pm 37.0 \mu \mathrm{m}, 140.0 \pm 33.0 \mu \mathrm{m}(\mathrm{P}=$ $0.157)$ and $137.0 \pm 29.0(\mathrm{P}=0.46)$, respectively. Nasal RNFL: $77.0 \pm 38.0 \mu \mathrm{m}, 84.0 \pm 26.0 \mu \mathrm{m}(\mathrm{P}=0.663)$, and $77.0 \pm 24.0 \mu \mathrm{m}(\mathrm{P}=0.75)$; inferior RNFL: $139.0 \pm$ $50.0 \mu \mathrm{m}, 137.0 \pm 44.0 \mu \mathrm{m}(\mathrm{P}=0.057)$, and $133.0 \pm$ $40.0 \mu \mathrm{m}(\mathrm{P}=0.23)$; temporal RNFL; $81.0 \pm 23.0 \mu \mathrm{m}$, $89.0 \pm 24.0 \mu \mathrm{m}(\mathrm{P}=0.017)$, and $87.0 \pm 27.0 \mu \mathrm{m}(\mathrm{P}=$ 0.06 ), at baseline and 2 and 4 months post-treatment, respectively (Table 2, Fig. 1 ).

Changes in average RNFL were only significant at 2 months post-treatment. Therefore, analysis was done using Kruskal-Wallis test looking at a possible association between the changes in RNFL and HbA1c levels. However, no significant association was found between change in RNFL thickness and the level of sugar control for the past 3 months. Mean rank for Group 1 (HbA1c <6.5\%) was 24.13; Group 2 (HbA1c of 6.6\%8.0\%) was 17.56; Group 3 (HbA1c of $8.1 \%-10.0 \%)$ was 27.56; and Group 4 (HbA1c $>10.1 \%$ ) was 15.22.

\section{Discussion}

This study demonstrated transient RNFL thickening in all quadrants, which occurred during the first two months after PRP. Significant changes were demonstrated for average RNFL. Although, other quadrants did not reach statistically significant differences, a consistent thickening trend was observed in all quadrants. Ritenour et al. reported, in his small study of 10 patients (8), thickening of RNFL (higher in the temporal quadrant) was also observed at 2 month post-laser and regression to baseline value occurred at 6 months post-treatment. Similar findings were described by Kim et al. in which significant transient thickening peaked at 1 month post-treatment with further gradual reduction of RNFL thickness after 12 months (9).This transient retinal thickening was most likely due to post-laser inflammatory effects. An animal study by Nonaka et al. suggested that PRP increases leucocyte rolling and aggravation in blood vessels. This leucocyte-endothelial reaction further contributes to the increase in retinal vessel permeability, which results in retinal swelling postlaser treatment (10).

Our study showed recovery of RNFL thickening fairly early compared to other studies $(9,11)$. At four months post-treatment, RNFL in all quadrants recovered to almost baseline values; there were no significant differences in any quadrants with respect to baseline thickness and four months post-treatment. In other studies, recovery of retinal oedema only occurs at six months $(9,11)$. Findings of further changes in the peripapillary RNFL during the latent phase of treatment are variable. For instance, a cross sectional study by Lim et al. reported significant thinning of the RNFL post-laser compared to the normal population (7). Lee et al. reported similar findings in their prospective longitudinal study conducted over two years (11).Conversely, Kim et al. did not find significant thinning in their study population over a 1 year follow-up; RNFL gradually reduced, but was not less than baseline values (9). We also found 
insignificant differences between baseline RNFL and the final thickness at the end of our study. We believe that a longer observation period will provide more valuable information regarding RNFL changes.

The objective and reproducible results of OCT imaging provides another platform for glaucoma assessment (12). Importantly, OCT has also been used to diagnose pre-perimetric glaucoma (13). Interestingly, Lim et al. highlighted that optic nerves post-PRP tend to be graded as abnormal although clinically they do not appear glaucomatous. Furthermore, they described a significant association between thin RNFL and clinically abnormal discs (7). Therefore, it is important for the clinician to be aware of conditions predisposing patients to abnormal RNFL thickness and evaluation of glaucoma must be made with caution.

In patients with diabetes, high blood HbA1c suggests poor blood sugar control, which might threaten tissue viability, which in turn theoretically makes the RNFL vulnerable to external insults, such as laser. However, this vulnerability did not translate into further damage to RNFL thickness post-laser treatment in our study. Poor diabetic control, as defined by HbA1c level, did not increase the risk of further RNFL changes immediately post-PRP. Interestingly, this association has never been highlighted in other studies and we believe that this should be further evaluated with more specific outcomes.

Limitations to this study include its short follow-up. However, within the study period, we managed to highlight the important observation of RNFL changes and relate our findings to many previous reports. A longer observation period will provide more details on the long term changes with relation to time. Secondly, time-domain OCT is an earlier version of tomography. Although the cellular detail is not as great as with the newer spectral domain OCT, RNFL thickness measurements using time-domain OCT are as good as the newer imaging techniques.

In conclusion, PRP causes transient thickening, particularly in the temporal RNFL. Therefore, the use of RNFL imaging for glaucoma diagnosis in this patient population must therefore be made with caution. At the same time, poor sugar control has no direct influence on the RNFL changes after PRP.

\section{Acknowledgement}

The authors acknowledge the help received from Dr. Zuraidah Che' Man from the Research Unit (CREM) and Dr. Azimatun from the Community Health Department for their statistical input.

\section{References}

1. Yanoff M, Duker JS. Ophthalmology. 4th ed. Oxford: Saunders, 2014, pp-542.

2. Early Treatment Diabetic Retinopathy Study Research Group. Early photocoagulation for diabetic retinopathy. ETDRS report number 9. Ophthalmology 1991; 98(5 Suppl): 766-85.

3. The Diabetic Retinopathy Study Research Group. Photocoagulation treatment of proliferative diabetic retinopathy. Clinical application of Diabetic Retinopathy Study (DRS) findings, DRS Report Number 8. Ophthalmol 1981; 88(7): 583600.

4. Rema M, Sujatha P, Pradeepa R. Visual outcomes of pan-retinal photocoagulation in diabetic retinopathy at one-year follow-up and associated risk factors. Indian $\mathrm{J}$ Ophthalmol 2005; 53(2): 93-9.

5. Kim HY, Cho HK. Peripapillary retinal nerve fiber layer thickness change after panretinal photocoagulation in patients with diabetic retinopathy. Korean J Ophthalmol 2009; 23(1): 23-6.

6. Yang Z, Tatham AJ, Zangwill LM, Weinreb RN, Zhang C, Medeiros FA. Diagnostic ability of retinal nerve fiber layer imaging by swept-source optical coherence tomography in glaucoma. Am J Ophthalmol 2015; 159(1): 193-201.

7. Lim MC, Tanimoto SA, Furlani BA, et al. Effect of diabetic retinopathy and panretinal photocoagulation on retinal nerve fiber layer and optic nerve appearance. Arch Ophthalmol 2009;127(7): 857-62.

8. Ritenour RJ, Kozousek V, Chauhan BC. The effect of panretinal photocoagulation for diabetic retinopathy on retinal nerve fibre layer thickness and optic disc topography. $\mathrm{Br} \mathrm{J}$ Ophthalmol 2009; 93(6): 838-9.

9. Kim JJ, Im JC, Shin JP, Kim IT, Park DH. Oneyear follow up of macular ganglion cell layer and peripapillary retinal nerve fibre layer thickness changes after panretinal photocoagulation. $\mathrm{Br} \mathrm{J}$ Ophthalmol 2014; 98(2): 213-7. 
10. Nonaka A, Kiryu J, Tsujikawa A, et al. Inflammatory response after scatter laser photocoagulation in non photocoagulated retina. Invest Ophthamol Vis Sci 2002; 43(4): 1204-9.

11. Lee SB, Kwag JY, Lee HJ, Jo YJ, Kim JY. The longitudinal changes of retinal nerve fiber layer thickness after panretinal photocoagulation in diabetic retinopathy patients. Retina 2013; 33(1): 188-93.

12. Paunescu LA, Schuman JS, Price LL, et al. Reproducibility of nerve fiber thickness, macular thickness, and optic nerve head measurement using Stratus OCT. Invest Ophthalmol Vis Sci 2004; 45(6): 1716-24.

13. Rao HL, Yadav RK, Addepalli UK, et al. Peripapillary retinal nerve fiber layer assessment of spectral domain optical coherence tomography and scanning laser polarimetry to diagnose preperimetric glaucoma. PLoS One 2014; 9(10): e108992. 